\title{
KINEMATICS OF MOLECULES AT THE GALACTIC CENTRE
}

\author{
B. J. ROBINSON \\ Division of Radiophysics, CSIRO, Sydney, Australia
}

\begin{abstract}
Dense gas clouds containing $\mathrm{OH}, \mathrm{CO}, \mathrm{NH}_{3}$ and $\mathrm{H}_{2} \mathrm{CO}$ are found in the inner part of the $\mathrm{H}_{1}$ nuclear disk. The molecular spectral lines allow direct observations of the kinematics of the gas near the galactic centre. Strong absorption of the thermal continuum sources by $\mathrm{OH}$ and $\mathrm{H}_{2} \mathrm{CO}$ shows that much of the gas on the near side of the centre can be located in a massive 'ring' expanding at $130 \mathrm{~km} \mathrm{~s}^{-1}$, which may have originated close to the nucleus about $10^{6} \mathrm{yr}$ ago. Observations of $\mathrm{CO}$ emission from beyond the centre show that the far side of the 'ring' is expanding at a lower velocity, less than $90 \mathrm{~km} \mathrm{~s}^{-1}$. Observations of $\mathrm{CO}$ and $\mathrm{NH}_{3}$ emission with positive velocities for $l<360^{\circ}$ are needed to establish whether the 'ring' is a continuous structure.

$\mathrm{OH}$ and $\mathrm{H}_{2} \mathrm{CO}$ are also observed to be falling towards the centre. There is no agreement as to the location of this infalling matter.

The nuclear regions of the Galaxy are compared with those of NGC 253, particularly in regard to expansional velocities, IR and radio emission, and $\mathrm{OH}$ absorption.
\end{abstract}

\section{Introduction}

The most direct observations of the galactic centre can be made in the infrared, in the radio continuum and recombination line emission, and by means of molecular spectral lines. At visual wavelengths our view is obscured by 27 mag of absorption in the dust found near the centre and in the intervening spiral arms. High-velocity hydrogen near the centre can be observed at $21 \mathrm{~cm}$ wavelength, but for $\mathrm{H}$ I with radial velocities between $\pm 50 \mathrm{~km} \mathrm{~s}^{-1}$ the central regions are blanketed by the wings of the $21-\mathrm{cm}$ emission from the spiral arms in front of and behind the centre.

The molecular spectral lines provide a particularly powerful probe of the central regions. The density of molecules there is several orders of magnitude greater than in the spiral arms, so that there is negligible confusion from gas in the intervening (or distant) arms. The high molecular densities are found in the inner parts of the "nuclear disk' inferred from $21 \mathrm{~cm}$ observations. The kinematics of the region are defined by the radial velocities of the four molecules which are widely distributed: hydroxyl, formaldehyde, carbon monoxide and ammonia. Many exotic molecules have been observed in the dense clouds near Sgr B2 (G0.7-0.0) and Sgr A (G0.0-0.0), but they have not been seen at other longitudes and so provide little kinematic information. Hydroxyl and formaldehyde are seen in absorption against the continuum sources near the centre, while carbon monoxide and ammonia appear in emission and so can be detected on the far side of the continuum sources.

\section{The Nuclear Disk}

High-velocity $21 \mathrm{~cm}$ emission is seen between longitudes $356^{\circ}<l<0^{\circ}$ with radial velocity $-250<V<-70 \mathrm{~km} \mathrm{~s}^{-1}$. This gas has been associated with a rapidly rotating 
nuclear disk extending to a radius $R$ of about $800 \mathrm{pc}$ from the galactic centre (Rougoor, 1964). For $l>0^{\circ}$ the distribution of the high-velocity $\mathrm{H}_{\mathrm{I}}$ is confused by emission from $\mathrm{HI}$ beyond the centre, and the structure of the disk has been inferred from models. The basic parameters of the disk derived by Oort (1971) and by Sanders and Wrixon (1973) are shown in Table I.

TABLE I

Parameters of the $\mathrm{H}$ I nuclear disk

\begin{tabular}{|c|c|c|}
\hline Parameter & $\begin{array}{l}\text { Oort } \\
(1971)\end{array}$ & $\begin{array}{l}\text { Sanders and Wrixon } \\
\text { (1973) }\end{array}$ \\
\hline Radius (pc) & 750 & 750 \\
\hline Thickness (pc) & $\begin{array}{l}100 \text { for } R<300 \mathrm{pc} \\
250 \text { for } R>300 \mathrm{pc}\end{array}$ & $\begin{array}{l}100 \text { for } 300<R<500 \mathrm{pc} \\
120 \text { on average }\end{array}$ \\
\hline Maximum rotational velocity $\left(\mathrm{km} \mathrm{s}^{-1}\right)$ & 230 & 230 \\
\hline Velocity dispersion $\left(\mathrm{km} \mathrm{s}^{-1}\right)$ & - & 13 \\
\hline H I density (atoms $\mathrm{cm}^{-3}$ ) & $\begin{array}{l}3 \text { for } R<100 \mathrm{pc} \\
0.3 \text { on average }\end{array}$ & $\begin{array}{l}5 \text { for } R<110 \mathrm{pc} \\
1.5 \text { on average }\end{array}$ \\
\hline Total H I mass $\left(M_{\odot}\right)$ & $4 \times 10^{6}$ & $2 \times 10^{6}$ for $R<500 \mathrm{pc}$ \\
\hline Dust mass $\left(M_{\odot}\right)$ & $10^{5}$ & - \\
\hline Mass of $\mathrm{H}_{2}\left(M_{\odot}\right)$ & $10^{7}$ & - \\
\hline
\end{tabular}

Oort's (1971) model is symmetric about the centre, with the gas increasing in density smoothly towards the centre and rotating in circular orbits. The model of Sanders and Wrixon (1973) is based on higher-resolution observations and has no neutral hydrogen in the inner $300 \mathrm{pc}$ on the positive longitude side of the disk; at negative longitudes the $\mathrm{HI}$ density increases smoothly to within $100 \mathrm{pc}$ of the centre.

We should bear in mind that the techniques which best probe the galactic centre all show a marked asymmetry about $l=0^{\circ}$. The $100 \mu \mathrm{m}$ IR observations (Hoffman et al., 1971) and the radio continuum observations (reviewed by Gordon in this volume) show a much greater extent (and strength) of the emission at positive longitudes than at negative longitudes. The molecules show an even more pronounced asymmetry.

\section{Distribution and Motions of $\mathrm{OH}$ Molecules}

The most complete survey of the distribution of a molecular species near the galactic centre has been made for $\mathrm{OH}$ in absorption at $18 \mathrm{~cm}$ wavelength (Robinson and McGee 1970; McGee et al., 1970). As shown in Figure 1, absorption extends in longitude from $359^{\circ}<l<2^{\circ}$ for $-180<V<+90 \mathrm{~km} \mathrm{~s}^{-1}$. Weaker absorption can be traced up to $l \approx 4^{\circ}$ at $b=0^{\circ}$, but considerable searching has failed to reveal $\mathrm{OH}$ absorption in the range $357^{\circ}<l<358^{\circ} 30^{\prime}$. At a distance of $10 \mathrm{kpc}, l=2^{\circ}$ corresponds to a radial distance of $350 \mathrm{pc}$ from the centre.

We see that the $\mathrm{OH}$ is concentrated in the inner part of the $\mathrm{HI}$ nuclear disk on the positive longitude side. The $\mathrm{OH}$ can be traced nearly to the edge of the disk, to $R=700 \mathrm{pc}$ (corresponding to $l=4^{\circ}$ ). Much of the $\mathrm{OH}$ has large radial motions towards 


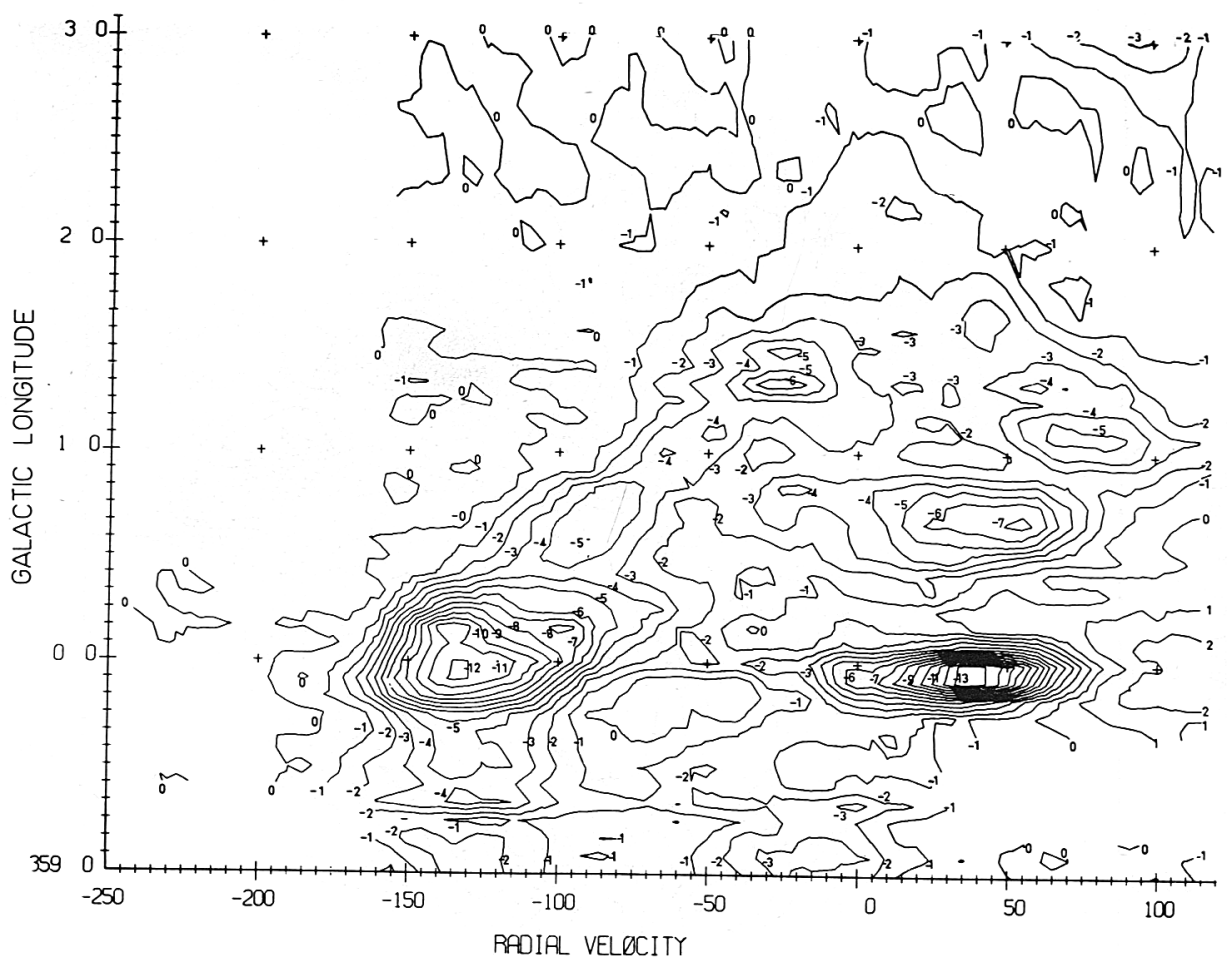

Fig. 1. Galactic longitude - radial velocity $(l-V)$ plot of $1667 \mathrm{MHz} \mathrm{OH}$ absorption for $b=-0^{\circ} 10^{\prime}$. Beamwidth is $12.2^{\prime}$, velocity resolution is $6.6 \mathrm{~km} \mathrm{~s}^{-1}$. Observations were made every $5^{\prime}$ in longitude. Contour unit is $1.7 \mathrm{~K}$ in antenna temperature. (From Robinson and McGee, 1970).

and away from the centre, unlike the circular motions adopted in the $\mathrm{H}$ i nuclear disk model.

The thickness of the hydroxyl layer for $R \leqslant 350 \mathrm{pc}$ is about 50 to $70 \mathrm{pc}$ (see McGee, 1970 ), compared to $100 \mathrm{pc}$ for the Hi disk. For $R>400 \mathrm{pc}$ the hydroxyl layer becomes somewhat thinner while the $\mathrm{H}$ I layer expands.

For $R \leqslant 350 \mathrm{pc}$ the hydroxyl absorption reaches a maximum about $10^{\prime}$ south of the galactic plane, corresponding to $z=-30 \mathrm{pc}$. No similar displacement is seen in the radio continuum or $100 \mu \mathrm{m}$ IR emission.

It might be argued that the asymmetry in longitude of the $\mathrm{OH}$ absorption simply reflects the asymmetry in distribution of the radio continuum. But McGee (1970) has shown that the asymmetry is even more marked in the distribution of apparent $\mathrm{OH}$ opacity. If we compare the apparent opacity in Figure 2 with the directly observed absorption in Figure 1, we see that the influence of strong continuum sources like Sgr A, Sgr B2 (G0.7-0.0) and G1.1-0.1 has been almost eliminated, and the highest opacity is found near $l=1^{\circ} 30^{\prime}$ and $l=3^{\circ}$.

Kaifu et al. (1972) drew attention to the continuity of the $\mathrm{OH}$ absorption in the $l-V$ plane shown by Figure 2, and suggested that the locus of the absorption was an ellipse as shown in Figure 3. Such a locus could be produced by a ring of $\mathrm{OH}$ with 


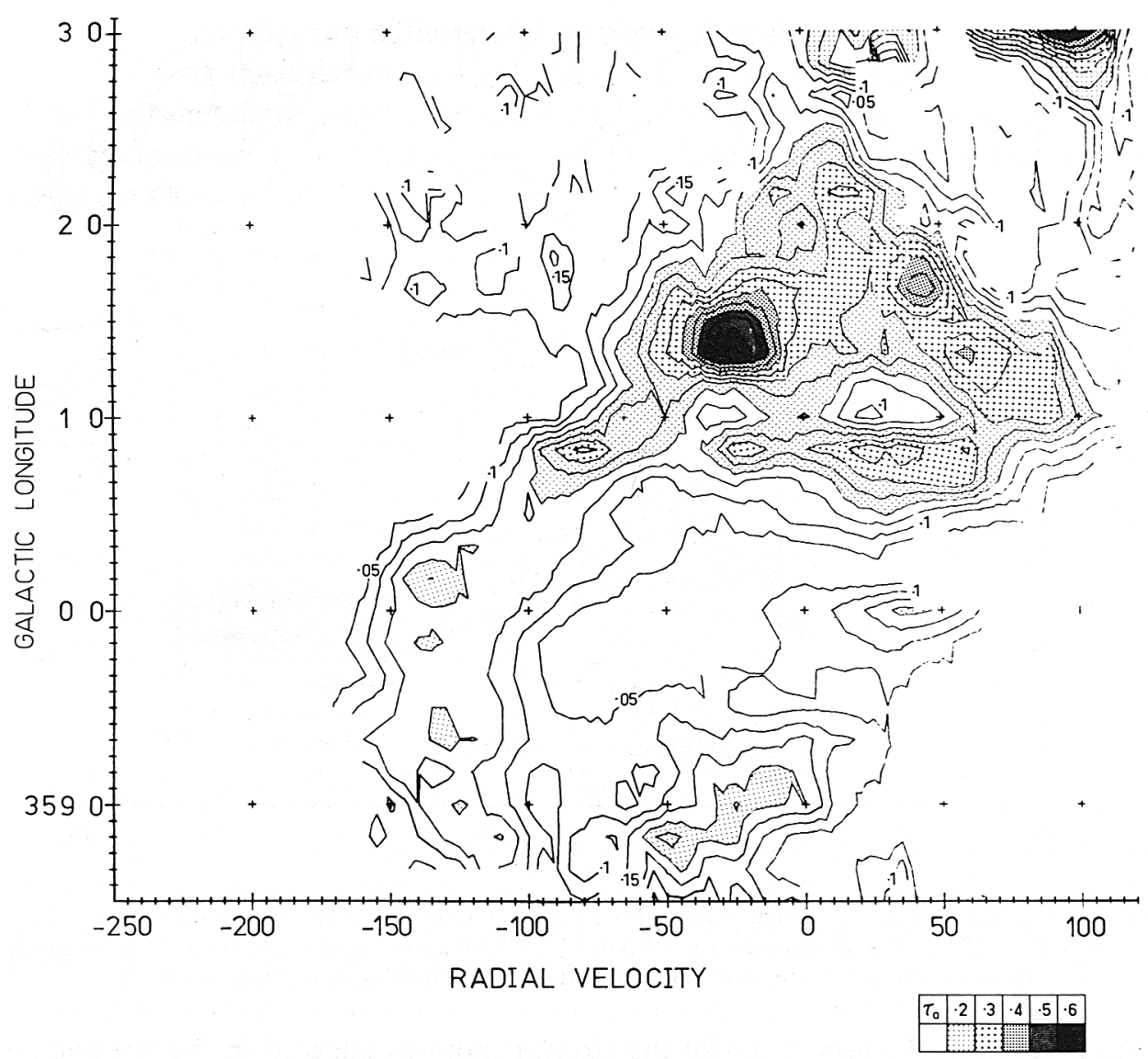

Fig. 2. Contours of apparent $\mathrm{OH}$ opacity at $1667 \mathrm{MHz}$ for $b=-0^{\circ} 10^{\prime}$, derived from Figure 1 . (From McGee, 1970).

$R=270 \mathrm{pc}$, rotating at $50 \mathrm{~km} \mathrm{~s}^{-1}$ and expanding at $130 \mathrm{~km} \mathrm{~s}^{-1}$. The positive velocity side of the ring is not observed, since the $\mathrm{OH}$ would be behind the continuum sources. However, Kaifu et al. showed that the velocities of some $\mathrm{NH}_{3}$ emission lines observed by Knowles and Cheung (1971) at $l=359^{\circ} 35^{\prime}$ and $l=1^{\circ} 12^{\prime}$ (marked by squares in Figure 3 ) fell on the locus “... thus confirming the existence of the expanding ring...". Models for this 'ring' will be discussed in a later section.

\section{Distribution and Motions of $\mathrm{H}_{2} \mathrm{CO}$ Molecules}

Absorption by the $1_{10} \leftarrow 1_{11}$ transition of formaldehyde at $6 \mathrm{~cm}$ has been surveyed by Gardner and Whiteoak (1970), Scoville et al. (1972) and Scoville and Solomon (1973). Figure 4 shows an $l-V$ contour diagram of the apparent opacity at $b=-0^{\circ} 12^{\prime}$. In general outline it shows marked similarities to the $\mathrm{OH}$ opacity in Figure 2, but more detail is apparent because of the four times higher resolution in velocity; the 


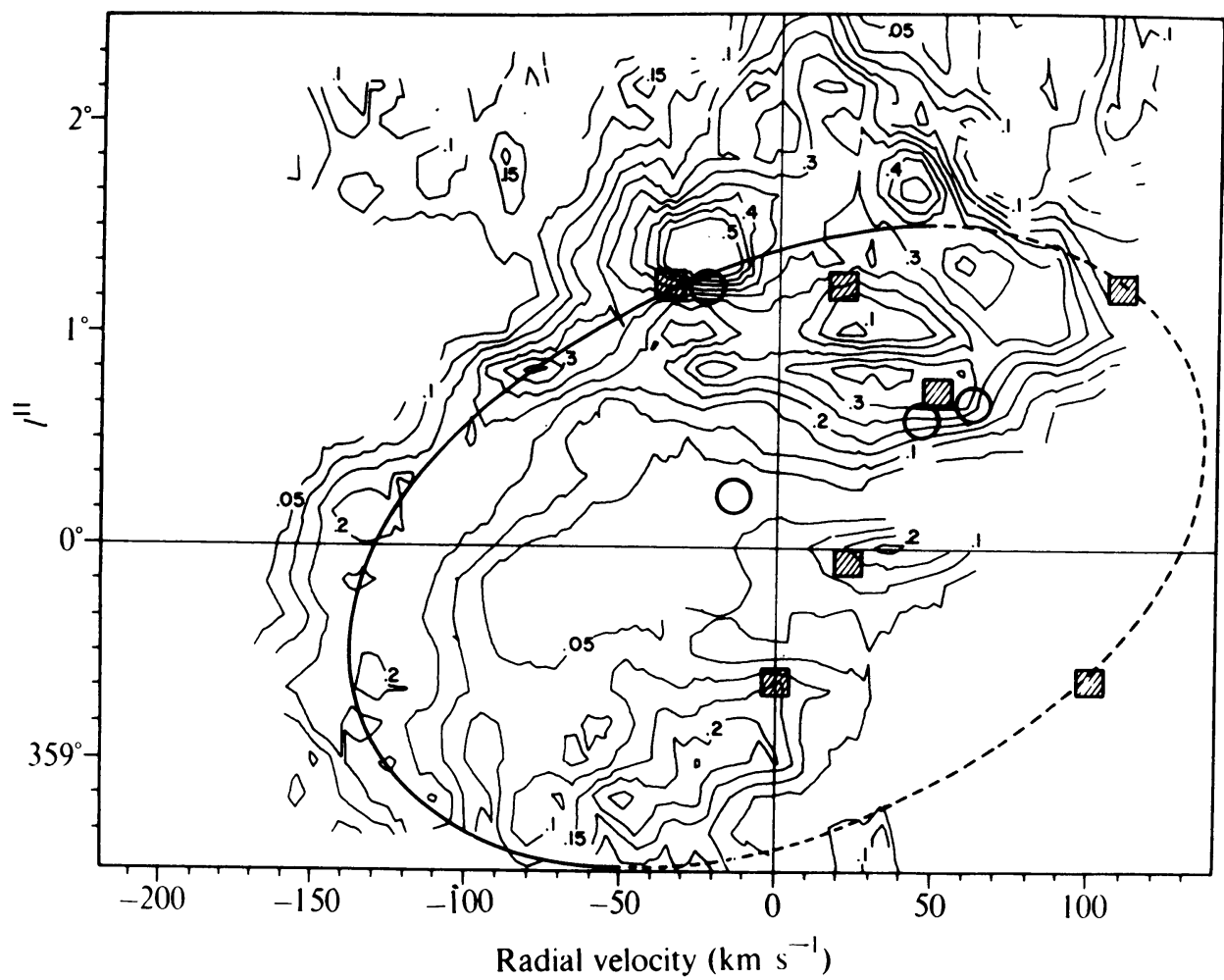

Fig. 3. Locus of $270 \mathrm{pc}$ expanding molecular ring suggested by Kaifu et al. (1972) from OH absorption (Figure 2) and $\mathrm{NH}_{3}$ emission velocities (shown by hatched rectangles). The circles indicate the hydrogen recombination line velocities of the $\mathrm{H}$ II regions near the galactic centre.

observations were undersampled in longitude. Narrow-band absorption by the $3 \mathrm{kpc}$ expanding arm (near $V=-50 \mathrm{~km} \mathrm{~s}^{-1}$ ) and in the nearby spiral arms (near $V=0 \mathrm{~km}$ $\mathrm{s}^{-1}$ ) is much more marked in the $\mathrm{H}_{2} \mathrm{CO}$ observations than for $\mathrm{OH}$. As was the case for $\mathrm{OH}$, the $\mathrm{H}_{2} \mathrm{CO}$ opacities are higher below the plane $\left(b=-0^{\circ} 12^{\prime}\right)$ than close to the plane $\left(b=-0^{\circ} 02^{\prime}\right)$; this is most marked for the negative velocity gas. $\mathrm{H}_{2} \mathrm{CO}$ observations at other latitudes have not been published.

Scoville (1972) showed that the maximum $\mathrm{H}_{2} \mathrm{CO}$ opacity also lies on part of an elliptical locus in the $l-V$ plane. This locus is shown by the continuous line in Figure 5, superimposed on contours of the average $\mathrm{H}_{2} \mathrm{CO}$ opacity at $b=-0^{\circ} 02^{\prime}$ and $b=-0^{\circ} 12^{\prime}$ The elliptical locus would correspond to a ring of gas of radius about $220 \mathrm{pc}$ rotating at $50 \mathrm{~km} \mathrm{~s}^{-1}$ and expanding at $145 \mathrm{~km} \mathrm{~s}^{-1}$; the ring extends further at positive longitudes $(R=305 \mathrm{pc})$ than at negative longitudes $(R=218 \mathrm{pc})$. As with the $\mathrm{OH}$ observations in Figure 3, data on the $\mathrm{H}_{2} \mathrm{CO}$ distribution at positive velocities are incomplete, presumably because the $\mathrm{H}_{2} \mathrm{CO}$ then lies beyond the continuum sources near the centre.

\section{Distribution and Motions of CO Molecules}

A preliminary $\mathrm{CO}$ emission line survey at $\lambda=2.6 \mathrm{~mm}$ covering a strip at $b=-0^{\circ} 02^{\prime}$ 
from $l=359.7$ to $l=2.8$ has been published by Solomon et al. (1972). As well as being restricted to positive longitudes the survey is also restricted to positive velocities $\left(+28<V<+130 \mathrm{~km} \mathrm{~s}^{-1}\right)$. The survey is also heavily undersampled in longitude, observations with a 1 arcmin beam being separated by $6^{\prime}$ (identical with the spacing used for $\mathrm{OH}$ and $\mathrm{H}_{2} \mathrm{CO}$ ).

The $l-V$ contour map for $\mathrm{CO}$ at $b=-0^{\circ} 02^{\prime}$ is shown in Figure 6. Between the centre and $l=18.8, \mathrm{CO}$ emission is observed over almost the complete velocity range of the measurements. The high-velocity CO features near $l=0^{\circ}\left(60<V<95 \mathrm{~km} \mathrm{~s}^{-1}\right)$, $l=0.7\left(80<V<105 \mathrm{~km} \mathrm{~s}^{-1}\right)$ and between these longitudes $\left(60<V<90 \mathrm{~km} \mathrm{~s}^{-1}\right)$ are extremely weak or missing from the $\mathrm{OH}$ and $\mathrm{H}_{2} \mathrm{CO}$ maps. The $\mathrm{CO}$ at these velocities is presumably beyond the centimetric continuum sources which are absorbed by $\mathrm{OH}$ and $\mathrm{H}_{2} \mathrm{CO}$.

At $l=1.4$ scans perpendicular to the plane showed that the $\mathrm{CO}$ emission extended beyond $b=+14^{\prime}$ and $b=-6^{\prime}$.

The $\mathrm{CO}$ observations indicate high densities and low temperatures in the molecular

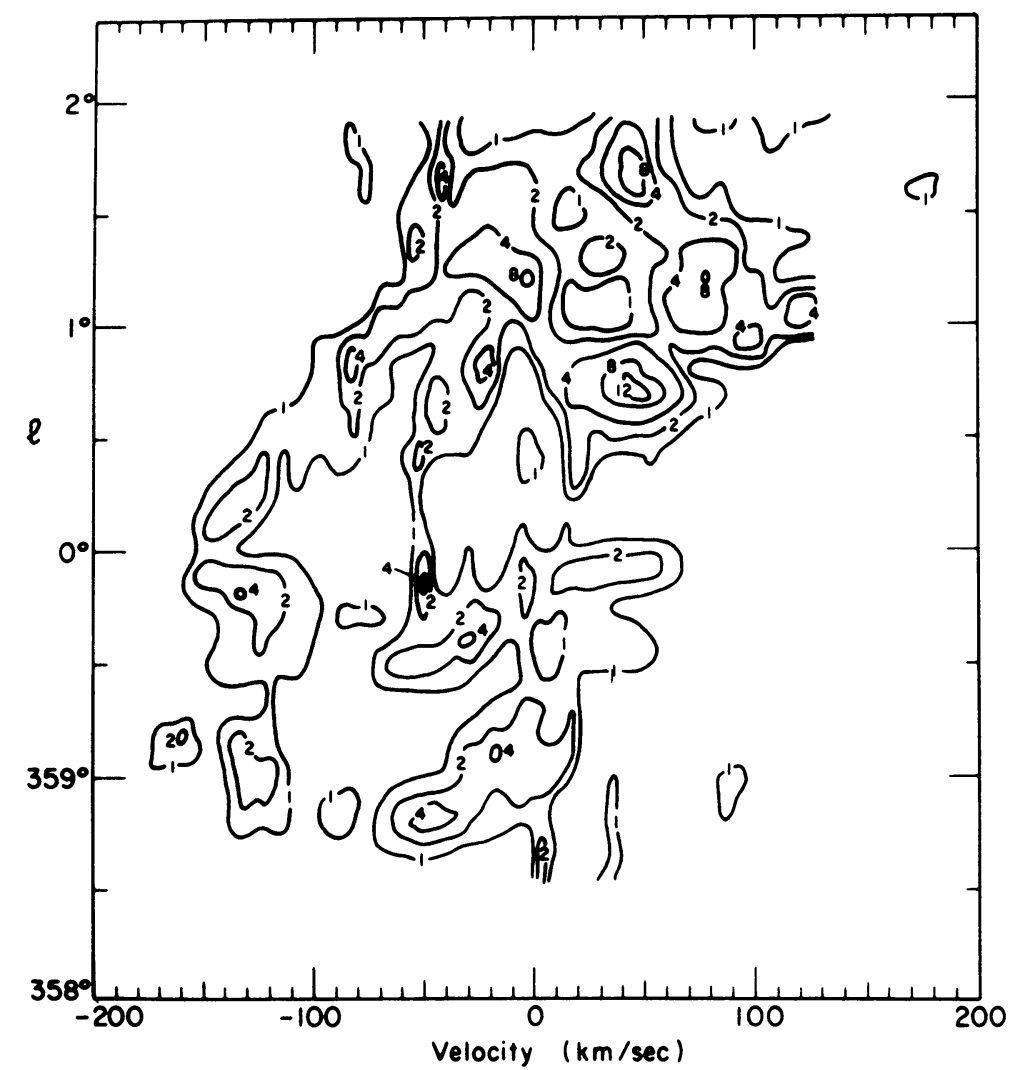

Fig. 4. Contours of apparent $\mathrm{H}_{2} \mathrm{CO}$ opacity at $4830 \mathrm{MHz}$ for $b=-0^{\circ} 12^{\prime}$. Beamwidth is $6.6^{\prime}$, velocity resolution is $1.63 \mathrm{~km} \mathrm{~s}^{-1}$. Observations were made every $6^{\prime}$ in longitude.

(From Scoville and Solomon, 1973). 


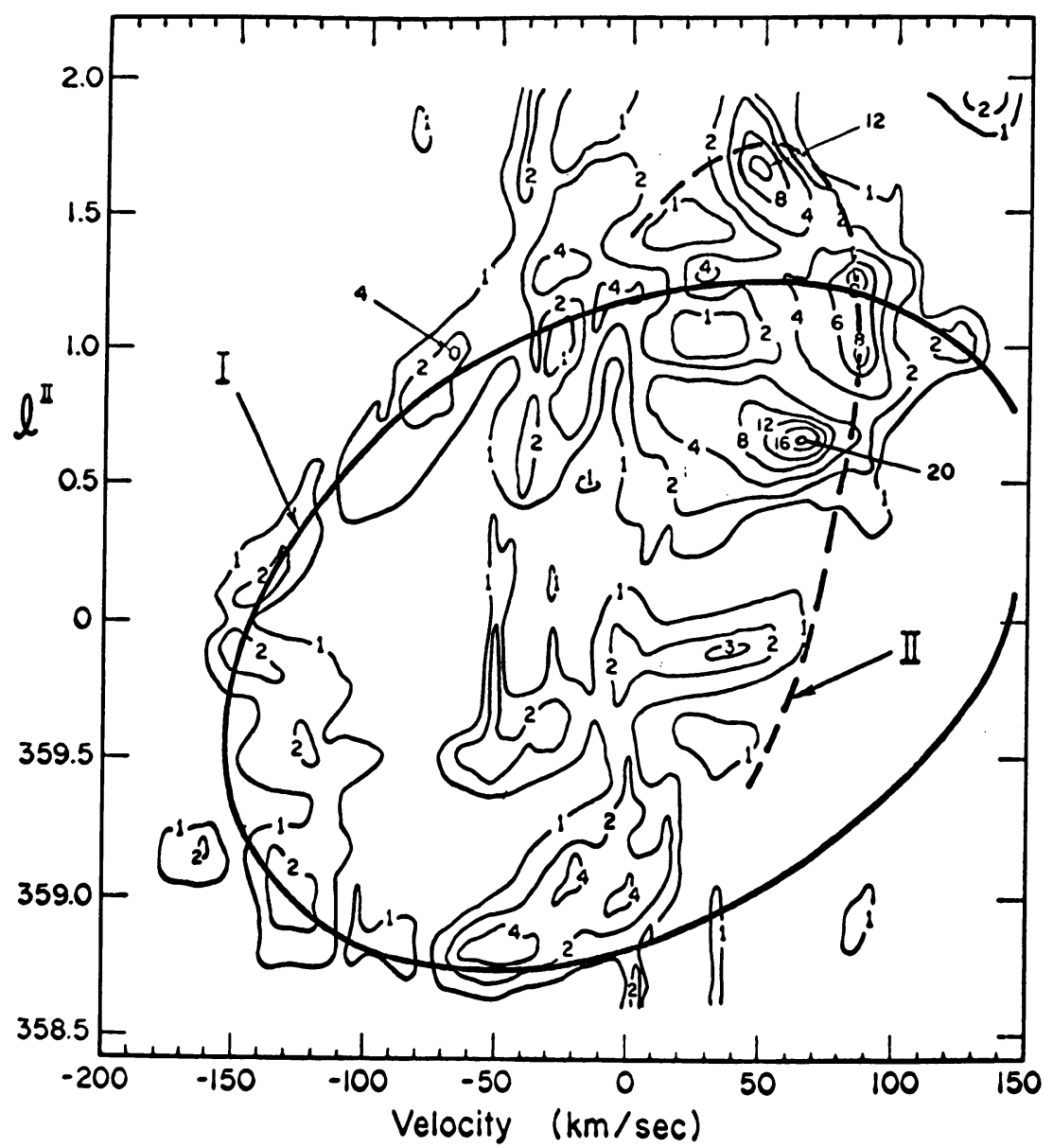

Fig. 5. Locus of expanding molecular ring suggested by Scoville (1972) from $\mathrm{H}_{2} \mathrm{CO}$ absorption. The $\mathrm{H}_{2} \mathrm{CO}$ opacity contours are the average of those at $b=-0^{\circ} 02^{\prime}$ and $b=-0^{\circ} 12^{\prime}$.

clouds. The upper state $(J=1)$ of the $2.6 \mathrm{~mm} \mathrm{CO}$ transition has a lifetime of $1.6 \times 10^{7} \mathrm{~s}$ and will be appreciably populated by collisions when the hydrogen density exceeds $10^{3}$ atoms and/or molecules per cubic centimetre. The observation of $\mathrm{CO}$ emission against the isotropic background radiation implies that the molecular clouds near the centre have hydrogen densities $\geqslant 10^{3} \mathrm{~cm}^{-3}$. A density exceeding $10^{3} \mathrm{~cm}^{-3}$ is also required to excite the $1.3 \mathrm{~cm}$ ammonia emission lines. The $21 \mathrm{~cm}$ model for the nuclear disk has $0.3<n_{\mathrm{H}}<5 \mathrm{~cm}^{-3}$, so the hydrogen in the clouds must be mainly in the molecular form.

Comparison of ${ }^{12} \mathrm{C}^{16} \mathrm{O}$ and ${ }^{13} \mathrm{C}^{16} \mathrm{O}$ lines shows that the ${ }^{12} \mathrm{CO}$ line is optically thick. Thus the observed brightness temperatures of 13 to $17 \mathrm{~K}$ are a measure of the kinetic temperature in the molecular clouds. Solomon et al. (1972) have pointed out that this temperature is close to the IR brightness temperature at $100 \mu \mathrm{m}$, suggesting 


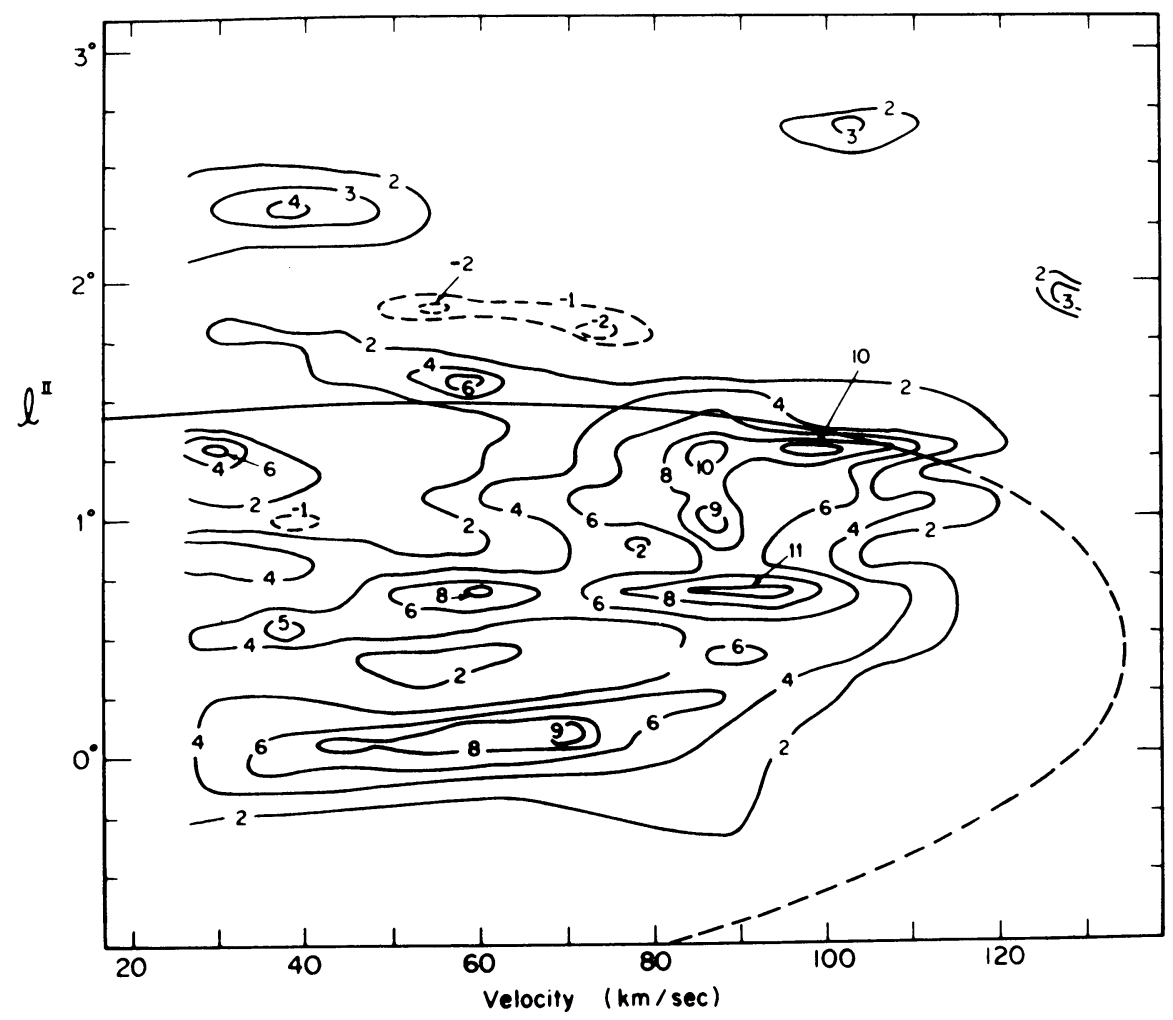

Fig. 6. Contours of $115 \mathrm{GHz} \mathrm{CO}$ emission for $b=-0^{\circ} 02^{\prime}$. Beamwidth is $1^{\prime}$, velocity resolution $5 \mathrm{~km} \mathrm{~s}^{-1}$. Observations were made every $6^{\prime}$ for $359^{\circ} 7<l<2^{\circ}$, every $12^{\prime}$ for $l>2^{\circ}$. The observations were restricted to $+28<V<+130 \mathrm{~km} \mathrm{~s}^{-1}$. (From Solomon et al., 1972). Part of the elliptical locus from Figure 3 is superimposed on the contours.

that the grains and gas are in thermal equilibrium and that the clouds are optically thick at $100 \mu \mathrm{m}$. This would imply $200 \mathrm{mag}$ of extinction at optical wavelengths.

\section{Models for the Molecular Ring}

The model for the expanding and rotating molecular ring derived by Kaifu et al. (1972) from $\mathrm{OH}$ absorption and $\mathrm{NH}_{3}$ emission is shown in Figure 7, and the parameters describing it are listed in Table II. The model proposed by Scoville (1972) from the $\mathrm{H}_{2} \mathrm{CO}$ absorption is shown in Figure 8, with the parameters also in Table II.

Kaifu et al. (1972) have noted that positive velocity $\mathrm{OH}$ and $\mathrm{H}_{2} \mathrm{CO}$ absorption is seen against Sgr A, Sgr B2 and other continuum sources near the center, and have put the infalling material in a smaller rotating and contracting ring with radius $140 \mathrm{pc}$. The recombination line velocities of the $\mathrm{H}$ il regions enable them to be located on this contracting ring (except for G1.1-0.1, which is located on the expanding ring). Scoville's (1972) model accounts for the positive velocity molecular absorption by 


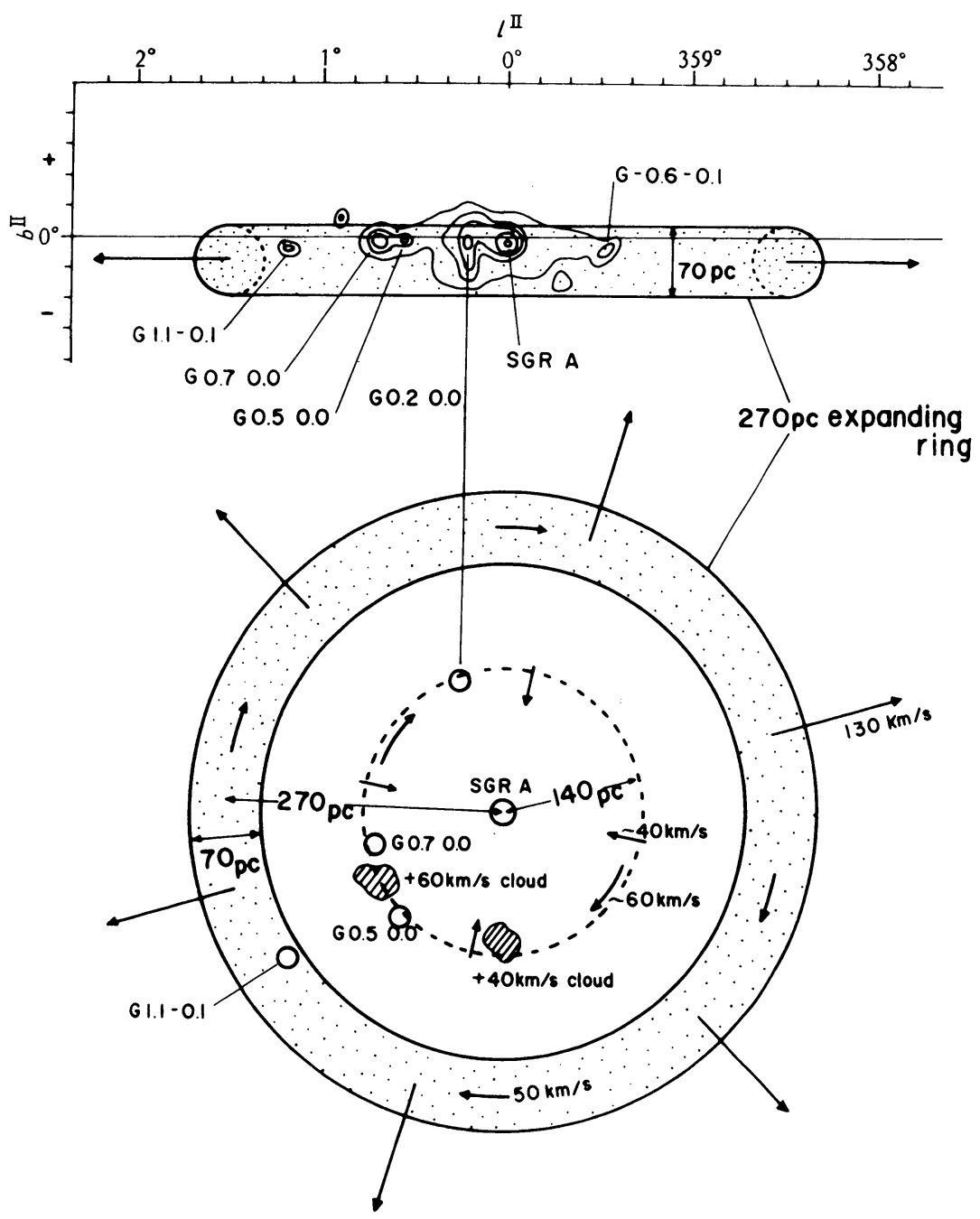

$100 \mathrm{pc}$

$\underbrace{}_{\text {Sun }}$

Fig. 7. Model for the $270 \mathrm{pc}$ expanding molecular ring proposed by Kaifu et al. (1972) from OH absorption and $\mathrm{NH}_{3}$ emission velocities. The broken circle shows the inner contracting ring, containing $\mathrm{OH}$ clouds (shaded patches) and $\mathrm{HI}$ regions (circles). The contours in the upper picture are $8 \mathrm{GHz}$ continuum contours. 
TABLE II

Parameters for the expanding molecular ring

\begin{tabular}{lll}
\hline Parameter & $\begin{array}{l}\text { Kaifu } \text { et al. } \\
(1972)\end{array}$ & $\begin{array}{l}\text { Scoville } \\
(1972)\end{array}$ \\
\hline Radius $(\mathrm{pc})$ & 270 & 218 \\
Thickness $\Delta z(\mathrm{pc})$ & 70 & - \\
Tilt to galactic plane & $6^{\circ}$ & 3 \\
Rotational velocity $\left(\mathrm{km} \mathrm{s}^{-1}\right)$ & 50 & 50 \\
Expansion velocity $\left(\mathrm{km} \mathrm{s}^{-1}\right)$ & 130 & 145 \\
OH column density $\left(\mathrm{cm}^{-2}\right)$ & $10^{16}$ & - \\
$\mathrm{H}_{2}$ CO column density $\left(\mathrm{cm}^{-2}\right)$ & $2 \times 10^{13}$ & - \\
$\mathrm{H}_{2}$ density $n_{\mathrm{H}_{2}}\left(\mathrm{~cm}^{-3}\right)$ & $\approx 10^{3}$ & $\leqslant 10^{3}$ \\
$\mathrm{H}$ density $n_{\mathrm{H}}\left(\mathrm{cm}^{-3}\right)$ & 2 & - \\
$n_{\mathrm{H}} / n_{\mathrm{H}_{2}}$ & $\approx 10^{-3}$ & - \\
Total mass of ring $\left(M_{\odot}\right)$ & $\approx 10^{8}$ & $\geqslant 10^{8}$ \\
Expansion time (years) & $2 \times 10^{6}$ & $\approx 10^{6}$ \\
Kinetic energy of expansion $(\mathrm{erg})$ & $\approx 10^{55}$ & $2 \times 10^{55}$ \\
\hline
\end{tabular}

putting all the $\mathrm{H}$ II regions and all but the nonthermal core of $\mathrm{Sgr} \mathrm{A}$ on the far side of the expanding ring. However, this disposition of the thermal sources is quite inconsistent with their recombination line velocities (see the points marked with circles on Figure 3).

To excite $\mathrm{CO}$ and $\mathrm{NH}_{3}$ emission the $\mathrm{H}_{2}$ density must exceed $10^{3} \mathrm{~cm}^{-3}$, and this makes the total mass of the expanding ring as high as $10^{8} M_{\odot}$. The kinetic energy of the expansion may exceed $10^{55} \mathrm{erg}$.

The angular velocity of the ring corresponds to that at a radius of about $50 \mathrm{pc}$ from the centre, so that the gas has probably expanded out from near the centre. At a constant speed of $135 \mathrm{~km} \mathrm{~s}^{-1}$ the expansion would have taken $2 \times 10^{6} \mathrm{yr}$. We do not

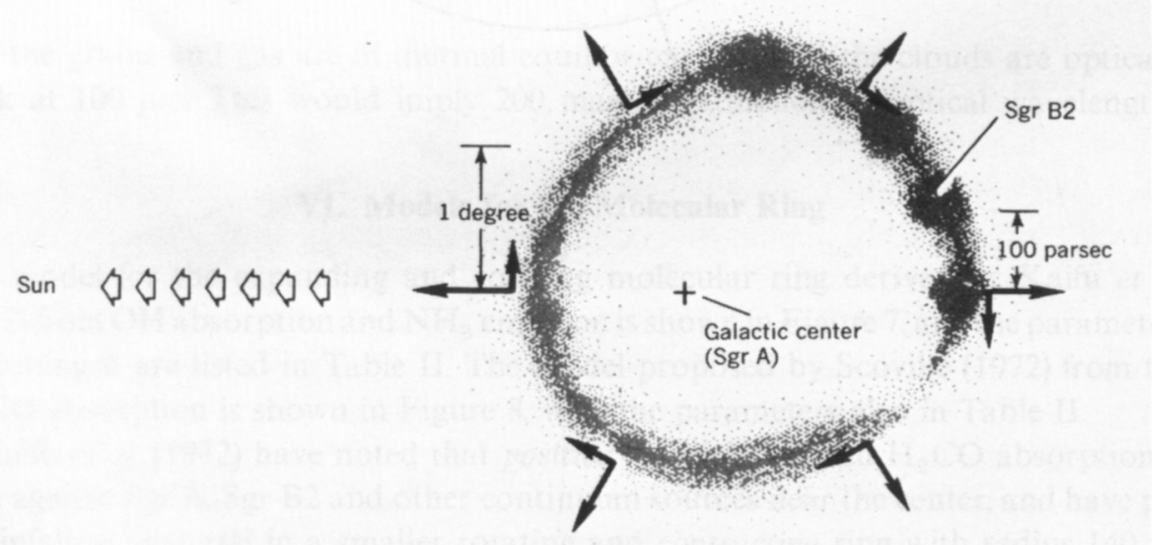

Fig. 8. Model for the expanding molecular ring proposed by Scoville (1972) from $\mathrm{H}_{2} \mathrm{CO}$ absorption velocities. The label Sgr A refers only to the nonthermal continuum component. The thermal continuum sources are located on the far side of the ring. 
understand what force could drive such an expansion and resort to an explanation in terms of 'an explosive event' in the nulceus about $10^{6} \mathrm{yr}$ ago. Sanders and Lowinger (1972) have computed circular velocities $V_{\mathrm{c}}$ in the nuclear disk; at $R=270 \mathrm{pc}$. $V_{\mathrm{c}}$ is about $230 \mathrm{~km} \mathrm{~s}^{-1}$ and so the escape velocity is about $330 \mathrm{~km} \mathrm{~s}^{-1}$. We see that the velocity of the expanding ring is far below the escape velocity. Thus unless there is some force to accelerate the ring the material will ultimately spiral back towards the nucleus.

Kaifu et al. (1972) note that the expansion velocity of $130 \mathrm{~km} \mathrm{~s}^{-1}$ far exceeds the sound velocity in the region ( 1 to $10 \mathrm{~km} \mathrm{~s}^{-1}$ ), and regard the expanding ring as a shock front. Strong compression by the shock leads to the formation of the observed molecular clouds and of stars and H II regions. Their contracting ring with $R=140 \mathrm{pc}$ may be a shock wave propagating towards the centre as the counter motion of gas after being swept by the outgoing shock wave (Kaifu et al., 1973).

We should take a critical look at the experimental data on which these models are based. Is the 'expanding ring' a continuous structure? Can we discriminate between a ring and an expanding spiral arm (or arms)? It is obvious from Figures 3, 5 and 6 that there is a serious lack of positive velocity data for $\mathrm{OH}, \mathrm{H}_{2} \mathrm{CO}$ and $\mathrm{CO}$ for $l<360^{\circ}$. Only one point appears in this quadrant, the $\mathrm{NH}_{3}$ velocity of $+100 \mathrm{~km} \mathrm{~s}^{-1}$ at $l=$ $359^{\circ} .35, b=-0.15$ (Figure 3). There is an urgent need for a survey of $\mathrm{CO}$ emission in this quadrant.

For $l>0^{\circ}$, the $\mathrm{CO}$ emission in Figure 6 for $80<V<120 \mathrm{~km} \mathrm{~s}^{-1}$ should arise beyond the centre, as little absorption at these velocities is seen for $\mathrm{OH}$ and $\mathrm{H}_{2} \mathrm{CO}$. However, when we draw the locus of the expanding ring (from Figure 3 ) on the $\mathrm{CO}$ contours in Figure 6, we see that the $\mathrm{CO}$ data give no support for the symmetric ring models in the part of the locus shown by the dashed line. The expansion velocity of the far side of the ring cannot be more than 90 or $100 \mathrm{~km} \mathrm{~s}^{-1}$.

\section{Similarity of the Nuclear Regions of the Galaxy and NGC 253}

Because high obscuration prevents any visual observations of the galactic centre it is fruitful to draw analogies with optical data on external galaxies. The popular model has been the nucleus of M31 (see Oort, 1971).

In M31 Rubin and Ford (1970) have found a striking asymmetry of the radial velocities near the nucleus; on the NE side the gas out to $R=800$ pc rotates at about the same velocity as the nuclear disk in the Galaxy; on the other side the motions are quite irregular. In the nucleus of M31 Sandage et al. (1969) found that the distribution of the $2.2 \mu \mathrm{m}$ IR radiation is identical with that at optical wavelengths; the $2.2 \mu \mathrm{m}$ distribution within $R=50 \mathrm{pc}$ is also identical with that in the Galaxy (Becklin and Neugebauer, 1968). This suggests that the $2.2 \mu \mathrm{m}$ distribution in the nucleus of the Galaxy can be used as a measure for the density distribution of the stars; within $R=20$ pc the stellar mass must be about $2 \times 10^{8} M_{\odot}$. Circular velocities computed for this stellar distribution are similar to those required for the $\mathrm{H}$ I nuclear disk.

An even better model for the nucleus of the Galaxy is provided by the nucleus of 
NGC 253. It is strikingly similar to the nucleus of the Galaxy in the IR, contains a nonthermal radio source similar to Sgr A, and contains a high density of $\mathrm{OH}$ molecules. Optical and $\mathrm{OH}$ observations reveal expansion from the nucleus.

A full comparison of the nuclei of the Galaxy and of NGC 253 is made in Table III. Expansion at a velocity of $120 \mathrm{~km} \mathrm{~s}^{-1}$ has been measured optically by Demoulin and Burbidge (1970) within $R=750 \mathrm{pc}$ of the nucleus of NGC 253. In addition, the first spiral arm at $R=3.5 \mathrm{kpc}$ is expanding at $V=40 \mathrm{~km} \mathrm{~s}^{-1}$, figures which are strikingly similar to those for our ' $3 \mathrm{kpc}$ expanding arm'.

TABLE III

Comparison of the nuclei of the Galaxy and NGC 253

\begin{tabular}{|c|c|c|}
\hline Property & Galactic centre & NGC 253 \\
\hline \multicolumn{3}{|l|}{ Expansion: } \\
\hline $\begin{array}{l}\text { At } R<750 \mathrm{pc} \\
\text { Of first spiral arm } \\
\text { (radius) }\end{array}$ & $\begin{array}{l}V_{\mathrm{E}}=130 \mathrm{~km} \mathrm{~s}^{-1} \\
V_{\mathrm{E}}=53 \mathrm{~km} \mathrm{~s}^{-1} \\
(R=4 \mathrm{kpc})\end{array}$ & $\begin{array}{l}V_{\mathrm{E}}=120 \mathrm{~km} \mathrm{~s}^{-1} \\
V_{\mathrm{E}}=40 \mathrm{~km} \mathrm{~s}^{-1} \\
(R=3.5 \mathrm{kpc})\end{array}$ \\
\hline \multicolumn{3}{|l|}{ IR source: } \\
\hline $\begin{array}{l}\text { Size } \\
\text { Luminosity }\end{array}$ & $\begin{array}{l}300 \mathrm{pc} \times 600 \mathrm{pc} \\
3.4 \times 10^{8} L_{\odot}\end{array}$ & $\begin{array}{l}150 \mathrm{pc} \times 150 \mathrm{pc} \\
3 \times 10^{9} L_{\odot}\end{array}$ \\
\hline \multicolumn{3}{|l|}{ Radio source: } \\
\hline Nonthermal radio energy & $3 \times 10^{50} \mathrm{erg}$ & $2 \times 10^{53} \mathrm{erg}$ \\
\hline \multicolumn{3}{|l|}{ Moleculés: } \\
\hline $\begin{array}{l}\mathrm{OH} \text { absorption }-V_{\text {expansion }} \\
\mathrm{OH} \text { masers (number) }\end{array}$ & $\frac{-130 \mathrm{~km} \mathrm{~s}^{-1}}{1}$ & $2^{-50 \mathrm{~km} \mathrm{~s}^{-1^{\mathrm{a}}}}$ \\
\hline
\end{tabular}

${ }^{a}$ Extends to $-150 \mathrm{~km} \mathrm{~s}^{-1}$.

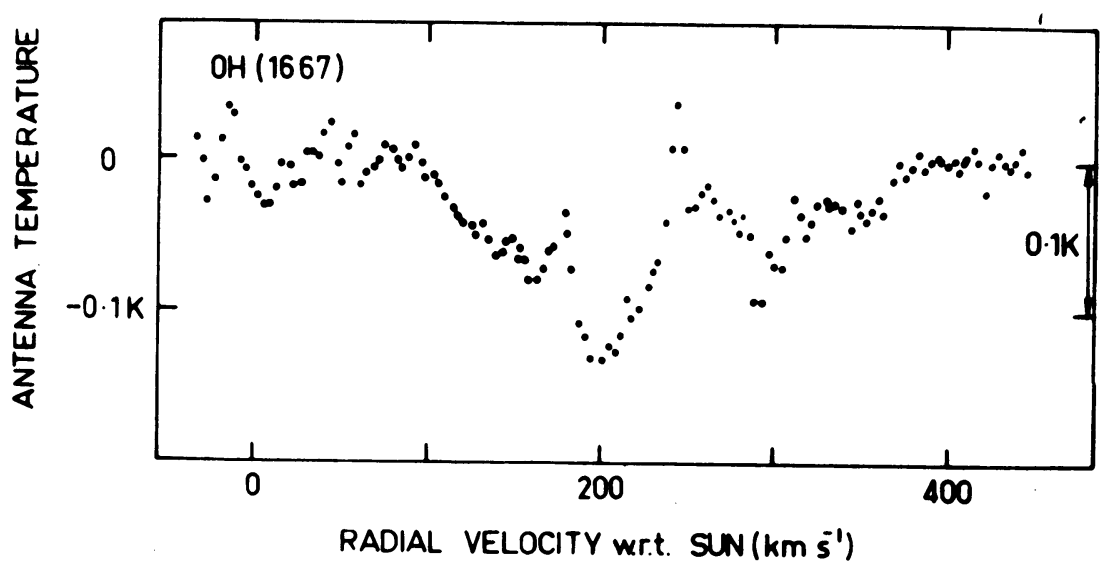

Fig. 9. $\mathrm{OH}$ absorption at $1667 \mathrm{MHz}$ of the nuclear radio source in NGC 253

(Whiteoak and Gardner, 1974). 
The far IR source in NGC 253 (Becklin et al., 1973) is somewhat smaller than the one at the centre of the Galaxy, but about ten times as luminous. The IR observations show that both nuclei have Seyfert characteristics. The nonthermal radio source in NGC 253 is nearly three orders of magnitude more energetic than Sgr A is at the present epoch.

Wideband $\mathrm{OH}$ absorption of the nonthermal radio source in NGC 253 is shown in Figure 9 (Whiteoak and Gardner, 1974). The systemic velocity is $250 \mathrm{~km} \mathrm{~s}^{-1}$, so that the strongest $\mathrm{OH}$ absorption corresponds to expansion at a velocity of $50 \mathrm{~km} \mathrm{~s}^{-1}$ there is less dense $\mathrm{OH}$ expanding at velocities up to $150 \mathrm{~km} \mathrm{~s}^{-1}$. Weaker $\mathrm{OH}$ absorption at velocities higher than the systemic velocity shows that there must also be infalling $\mathrm{OH}$ with $V \approx 50 \mathrm{~km} \mathrm{~s}^{-1}$. Two narrow spikes on the profile may be $\mathrm{OH}$ maser sources. In all, the $\mathrm{OH}$ profile in Figure 9 is strikingly similar to the $\mathrm{OH}$ profile of Sgr B2 (see Robinson et al., 1970, Figure 7).

\section{Conclusions}

Observations of hydroxyl, formaldehyde, carbon monoxide and ammonia lines near the galactic centre provide evidence for a section of a dense ring of matter about $270 \mathrm{pc}$ in radius, expanding at about $130 \mathrm{~km} \mathrm{~s}^{-1}$ (see Table II). The ring must have a mass of about $10^{8} M_{\odot}$. It lies well within the rapidly rotating HI nuclear disk (see Table I); few molecules are seen in the outer parts of the nuclear disk, or inside the radius of the molecular ring.

The molecular ring has a rotational velocity of $50 \mathrm{~km} \mathrm{~s}^{-1}$ and so does not share the rotation of the $\mathrm{HI}$ nuclear disk - which would be about $230 \mathrm{~km} \mathrm{~s}^{-1}$ at $R=270 \mathrm{pc}$. The expansion velocity of the ring is well below the escape velocity at $R=270 \mathrm{pc}$, which would be about $340 \mathrm{~km} \mathrm{~s}^{-1}$.

Kaifu et al. (1972) have suggested that the molecular ring has been produced by a shock wave travelling outwards from about $100 \mathrm{pc}$ from the centre. The strong compression produced by the shock wave would lead to the formation of molecules and of young stars and $\mathrm{H}$ II regions. Since the expansion time of the ring would be about $2 \times 10^{6} \mathrm{yr}$, this model implies that the formation time for the molecules is about $10^{5}$ to $10^{6} \mathrm{yr}$. This requires high densities. High densities are also indicated by the excitation of $\mathrm{CO}$ and $\mathrm{NH}_{3}$ to emit.

The observations of $\mathrm{OH}$ and $\mathrm{H}_{2} \mathrm{CO}$ show that material is also falling in towards the galactic centre. Kaifu et al. (1972) locate these positive velocity molecular clouds on an inner ring of radius about $140 \mathrm{pc}$, contracting inwards at $V=40 \mathrm{~km} \mathrm{~s}^{-1}$ and rotating at $50 \mathrm{~km} \mathrm{~s}^{-1}$. The recombination line velocities of many of the $\mathrm{HII}$ regions allow them to be located on this inner ring. The inner ring is perhaps a counter shock produced by particles spiralling back towards the nucleus under its gravitational attraction. Scoville (1972) places the positive velocity molecular clouds and the HII regions on the far side of the $270 \mathrm{pc}$ expanding ring, but this location conflicts with the observed recombination line velocities.

Observational data about the far side of the $270 \mathrm{pc}$ expanding ring are very limited. 
The CO data for $l>0^{\circ}$ do not support the elegant symmetry of the ring proposed by Kaifu et al. (1972) and Scoville (1972). Observations of $\mathrm{CO}$ and $\mathrm{NH}_{3}$ emission with positive velocities for $l<360^{\circ}$ are badly needed to define the structure of the ring, or to test whether it may be an expanding spiral arm (or arms).

Finally, attention is drawn to the strong similarities between the nuclei of the Galaxy and of NGC 253 in the infrared, radio continuum and OH absorption. Optical and $\mathrm{OH}$ velocities for NGC 253 show similar expanding and contracting motions to those found at the centre of the Galaxy.

\section{References}

Becklin, E. E. and Neugebauer, G.: 1968, Astrophys. J. 151, 145.

Becklin, E. E., Fomalont, E. B., and Neugebauer, G.: 1973, Astrophys. J. Letters 181, L27.

Demoulin, M. H. and Burbidge, E. M.: 1970, Astrophys. J. 159, 799.

Gardner, F. F. and Whiteoak, J. B.: 1970, Astrophys. Letters 5, 161.

Hoffmann, W. F., Frederick, C. L., and Emery, R. L.: 1971, Astrophys. J. Letters 164, L23.

Kaifu, N., Iguchi, T., and Kato, T.: 1974, Publ. Astron. Soc. Japan 26, 117.

Kaifu, N., Kato, T., and Iguchi, T.: 1972, Nature 238, 105.

Knowles, S. H. and Cheung, A. C.: 1971, Astrophys. J. Letters 164, L19.

McGee, R. X.: 1970, Australian J. Phys. 23, 541.

McGee, R. X., Brooks, J. W., Sinclair, M. W., and Batchelor, R. A.: 1970, Australian J. Phys. 23, 777.

Oort, J. H.: 1971, in D. J. K. O'Connell (ed.), Nuclei of Galaxies, Pontificiae Academiae Scientiarum

Scripta Varia, North-Holland Publishing Co., Amsterdam, p. 321.

Robinson, B. J. and McGee, R. X.: 1970, Australian J. Phys. 23, 405.

Robinson, B. J., Goss, W. M., and Manchester, R. N.: 1970, Australian J. Phys. 23, 363.

Rougoor, G. W.: 1964, Bull. Astron. Inst. Neth. 17, 381.

Rubin, V. C. and Ford, W. K. : 1970, Astrophys. J. $159,379$.

Sandage, A. R., Becklin, E. E., and Neugebauer, G.: 1969, Astrophys. J. 157, 55.

Sanders, R. H. and Lowinger, T. L.: 1972, Astron. J. 77, 292.

Sanders, R. H. and Wrixon, G. T.: 1973, Astron. Astrophys. 26, 365.

Scoville, N. Z.: 1972, Astrophys. J. Letters 175, L127.

Scoville, N. Z. and Solomon, P. M.: 1973, Astrophys. J. 180, 55.

Scoville, N. Z., Solomon, P. M., and Thaddeus, P.: 1972, Astrophys. J. 172, 335.

Solomon, P. M., Scoville, N. Z., Jefferts, K. B., Penzias, A. A., and Wilson, R. W.: 1972, Astrophys. J. 178, 125.

Whiteoak, J. B. and Gardner, F. F.: 1974, Astrophys. Letters, in press; Nature 247, 526.

\section{B. J. Robinson}

CSIRO Division of Radiophysics, P.O. Box 76,

Epping, N.S.W. 2121, Australia

\section{DISCUSSION}

Morimoto: Shock wave calculations by Kaifu and others show that a secondary shock traveling outward with particles moving inward (thus appearing as a contracting ring) can develop as the particles behind the expanding ring fall back to the equilibrium position. This can explain the positive velocity features. The place where you left the symmetrical ellipse for an expanding ring in the $l, v$ diagram is where confusion from the contracting ring is the heaviest.

Robinson: At the outer edges of the ring, the galactic rotation curve deduced from the infrared observations gives circular velocities between 200 and $250 \mathrm{~km} \mathrm{~s}^{-1}$. The rotational velocity in the ring is on the 
order of $50 \mathrm{~km} \mathrm{~s}^{-1}$. If it has been blown out from the center, it started much closer in and has angular momentum comparable with that distance much closer in. There is then a problem of how it is going to stay up there without spiraling back into the center.

Morimoto: Matter is just starting to go back in.

Caswell: If they are real, how strong are the maser emission sources in NGC 253 compared with the strongest ones in our own Galaxy?

Gardner: It is somewhat more than 10 times greater than W49.

Batchelor: To what extent is the southern velocity quadrant of the $\mathrm{OH}$ map influenced by the continuum background?

Robinson: We worked very hard to get absorption down there at $18 \mathrm{~cm}$. The $\mathrm{CO}$ observations are not affected by the continuum, so you should see CO, but it certainly doesn't show up on the map.

Zuckerman: Those masers in NGC 253 must be really strong, since that was at $1667 \mathrm{MHz}$ and the strongest galactic masers are at $1665 \mathrm{MHz}$.

Robinson: It is comparable with the $1667 \mathrm{MHz}$ emission from G330.9-0.4.

Zuckerman: Those southern sources !

Robinson: You'll have to take our word for it that they exist. 